\title{
Cyclic Leibniz rule: a formulation of supersymmetry on lattice
}

\section{Mitsuhiro Kato}

Institute of Physics, University of Tokyo, Komaba, Meguro-ku, Tokyo 153-8902, Japan

E-mail: kato@hep1.c.u-tokyo.ac.jp

\section{Makoto Sakamoto}

Department of Physics, Kobe University, Nada-ku, Hyogo 657-8501, Japan

E-mail: dragonakobe-u.ac.jp

\section{Hiroto So*†}

Department of Physics, Ehime University, Bunkyou-chou 2-5, Matsuyama 790-8577, Japan

E-mail: so@phys.sci.ehime-u.ac.jp

For the purpose of constructing supersymmetric(SUSY) theories on lattice, we propose a new type relation on lattice -cyclic Leibniz rule(CLR)- which is slightly different from an ordinary Leibniz rule. Actually, we find that CLR can enlarge the number of SUSYs and construct more Nicolai mappings in a quantum-mechanical model. In this model, the exact mass degeneracy between fermion and boson is shown.

31st International Symposium on Lattice Field Theory LATTICE 2013

July 29 - August 3, 2013

Mainz, Germany

\footnotetext{
${ }^{*}$ Speaker.

$\dagger$ This work was supported in part by Grants-in-Aid for Scientific Research (No.25400260 and No.25287049) by the Japanese Ministry of Education, Science, Sports and Culture.
} 


\section{Motivations}

One of purposes of constructing lattice supersymmetry(SUSY) is to obtain a method of its nonperturbative analysis. Although the exact lattice supersymmetry is necessary for the analysis, there is an obstruction: Leibniz rule on lattice theories with locality [1 2]. The anticommutator between supercharges includes an infinitesimal translation and its fact makes the realization of its lattice framework with locality difficult. The realization of Leibniz rule with locality on lattice is one of the difficulties. Recently, we found an alternative of the rule on lattice [3]. It is called cyclic Leibniz rule(CLR). The new rule is applicable to local lattice theories with a subset of full supersymmetry. This subset symmetry is nilpotent and the algebra does not include translation operator. To apply the symmetry for particle physics, the mass degeneracy between fermions and bosons, and the stability against quantum fluctuations such as the effective action are very important. We have to recognize that the subset symmetry keeps the degeneracy. The stable property is commented in the summary section. In this talk, we shall show the realization of the exact symmetry for a quantum mechanical model. In the model, we explicitly show that fermion's and boson's masses are exactly degenerate and there exist more conserved supercharges and Nicolai mappings than the previous work's results [4, 5].

\section{A key relation:cyclic Leibniz rule}

The setting of one-dimensional lattice systems is explained. The lattice size is denoted by $N_{L}$ and an integer $n$ is used as the lattice site labeling and the lattice constant $a$ is set to unity. Higher dimensional extensions are discussed in the last section.

We write a general difference operator for a field $\phi_{n}$ as $(\Delta \phi)_{n}$

$$
(\Delta \phi)_{m} \equiv \sum_{n} \Delta_{m n} \phi_{n}
$$

and the symmetric field product rule on two fields $\phi_{n}$ and $\psi_{n}$ as $\{\phi, \psi\}_{\ell}^{M}$

$$
\{\phi, \psi\}_{\ell}^{M} \equiv \sum_{m n} M_{\ell m n} \phi_{m} \psi_{n}=\sum_{m n} M_{\ell n m} \phi_{m} \psi_{n}
$$

Introducing an inner product on a lattice space

$$
(\phi, \psi) \equiv \sum_{n} \phi_{n} \psi_{n}
$$

a usual Leibniz rule on lattice can be expressed as

$$
\left(\chi, \Delta\{\phi, \psi\}^{M}\right)=\left(\chi,\{\Delta \phi, \psi\}^{M}\right)+\left(\chi,\{\phi, \Delta \psi\}^{M}\right),
$$

for any three bosonic fields, $\phi_{n}, \psi_{n}, \chi_{n}$. If we take a generalized symmetric difference operator $\Delta=-\Delta^{T}$, the rule is expressed as

$$
\left(\Delta \chi,\{\phi, \psi\}^{M}\right)+\left(\chi,\{\Delta \phi, \psi\}^{M}\right)+\left(\chi,\{\phi, \Delta \psi\}^{M}\right)=0
$$


On the other hand, we present a new alternative relation on a set of a difference operator and a symmetric product rule; that is cyclic Leibniz rule(CLR) [3]

$$
\left(\Delta \chi,\{\phi, \psi\}^{M}\right)+\left(\Delta \phi,\{\psi, \chi\}^{M}\right)+\left(\Delta \psi,\{\chi, \phi\}^{M}\right)=0 .
$$

While the Leibniz rule cannot be realized on lattice together with locality and translational invariance, this CLR can be realized locally. Although there are many solutions for CLR, we here give a simple example

$$
\Delta_{m n}=\frac{\delta_{m+1, n}-\delta_{m-1, n}}{2}, M_{\ell m n}=\frac{2 \delta_{\ell+1, m} \delta_{\ell+1, n}+2 \delta_{\ell-1, m} \delta_{\ell-1, n}+\delta_{\ell-1, m} \delta_{\ell+1, n}+\delta_{\ell+1, m} \delta_{\ell-1, n}}{6} .
$$

In fact, This example 2.77 is realized with locality and translational invariance. This CLR is sufficient to keep a subset of supersymmetry. If we extend to full supersymmetry, then we encounter the Leibniz rule and it cannot be realized in the local lattice formulation.

\section{Supersymmetric complex quantum mechanics}

To construct $N=2$ supersymmetry in one-dimensional lattice systems, we set the matter content of this model; for complex bosonic fields, $\phi_{+n}, F_{+n}$, their complex conjugates, $\phi_{-n}, F_{-n}$ and for fermionic fields, $\chi_{ \pm n}, \bar{\chi}_{ \pm n}$. For these fields, $N=2$ supersymmetry transformation

$$
\begin{aligned}
& \delta_{+} \phi_{+n}=\bar{\chi}_{+n}, \\
& \delta_{-} \phi_{+n}=0, \\
& \bar{\delta}_{+} \phi_{+n}=0 \\
& \bar{\delta}_{-} \phi_{+n}=-\chi_{+n} \\
& \delta_{+} \phi_{-n}=0 \text {, } \\
& \delta_{-} \phi_{-n}=\bar{\chi}_{-n}, \\
& \bar{\delta}_{+} \phi_{-n}=-\chi_{-n}, \\
& \bar{\delta}_{-} \phi_{-n}=0 \text {, } \\
& \delta_{+} \bar{\chi}_{+n}=0 \text {, } \\
& \delta_{-} \bar{\chi}_{+n}=0 \text {, } \\
& \bar{\delta}_{+} \bar{\chi}_{+n}=-i\left(\Delta \phi_{+}\right)_{n}, \\
& \bar{\delta}_{-} \bar{\chi}_{+n}=F_{+n}, \\
& \delta_{+} \chi_{+n}=F_{+n}, \\
& \delta_{-} \chi_{+n}=i\left(\Delta \phi_{+}\right)_{n}, \\
& \bar{\delta}_{+} \bar{\chi}_{+n}=0 \text {, } \\
& \bar{\delta}_{-} \chi_{+n}=0 \text {, } \\
& \delta_{+} \bar{\chi}_{-n}=0 \text {, } \\
& \delta_{-} \bar{\chi}_{-n}=0 \text {, } \\
& \bar{\delta}_{+} \bar{\chi}_{-n}=F_{-n}, \\
& \bar{\delta}_{-} \chi_{-n}=-i\left(\Delta \phi_{-}\right)_{n} \text {, } \\
& \delta_{+} \chi_{-n}=i\left(\Delta \phi_{-}\right)_{n}, \\
& \delta_{-} \chi_{-n}=F_{-n}, \\
& \bar{\delta}_{+} \chi_{-n}=0, \\
& \bar{\delta}_{-} \chi_{-n}=0 \text {, } \\
& \delta_{+} F_{+n}=0 \text {, } \\
& \delta_{-} F_{+n}=-i\left(\Delta \bar{\chi}_{+}\right)_{n}, \\
& \bar{\delta}_{+} F_{+n}=-i\left(\Delta \chi_{+}\right)_{n}, \\
& \bar{\delta}_{-} F_{+n}=0 \text {, } \\
& \delta_{+} F_{-n}=-i\left(\Delta \bar{\chi}_{-}\right)_{n}, \\
& \delta_{-} F_{-n}=0 \text {, } \\
& \bar{\delta}_{+} F_{-n}=0, \\
& \bar{\delta}_{-} F_{-n}=-i\left(\Delta \chi_{-}\right)_{n} \text {, }
\end{aligned}
$$

is defined. The algebra

$$
\left\{\boldsymbol{\delta}_{ \pm}, \boldsymbol{\delta}_{ \pm}\right\}=\left\{\overline{\boldsymbol{\delta}}_{ \pm}, \overline{\boldsymbol{\delta}}_{ \pm}\right\}=\left\{\boldsymbol{\delta}_{ \pm}, \boldsymbol{\delta}_{\mp}\right\}=\left\{\overline{\boldsymbol{\delta}}_{ \pm}, \overline{\boldsymbol{\delta}}_{\mp}\right\}=0 .
$$

follows and the remaining algebra

$$
\left\{\overline{\boldsymbol{\delta}}_{+}, \boldsymbol{\delta}_{+}\right\}+\left\{\overline{\boldsymbol{\delta}}_{-}, \boldsymbol{\delta}_{-}\right\}=-2 i \Delta
$$

is broken by $O(a)$ since this $\Delta$ is dissatisfied with a Leibniz rule[1]. By using CLR, we shall construct exactly supersymmetric complex quantum mechanics on lattice. The supersymmetric action is

$$
\begin{aligned}
S= & \left(\Delta \phi_{-}, \Delta \phi_{+}\right)-i\left(\Delta \bar{\chi}_{-}, \chi_{+}\right)-i\left(\Delta \bar{\chi}_{+}, \chi_{-}\right)+\left(F_{-}, F_{+}\right) \\
& +i\left(\phi_{+}, m F_{+}\right)+i\left(\bar{\chi}_{+}, m \chi_{+}\right)+i\left(\phi_{-}, m^{*} F_{-}\right)+i\left(\bar{\chi}_{-}, m^{*} \chi_{-}\right) \\
& +i \lambda\left(\left\{\phi_{+}, \phi_{+}\right\}^{M}, F_{+}\right)+2 i \lambda\left(\left\{\bar{\chi}_{+}, \phi_{+}\right\}^{M}, \chi_{+}\right) \\
& +i \lambda^{*}\left(\left\{\phi_{-}, \phi_{-}\right\}^{\bar{M}}, F_{-}\right)+2 i \lambda^{*}\left(\left\{\bar{\chi}_{-}, \phi_{-}\right\}^{\bar{M}}, \chi_{-}\right)
\end{aligned}
$$


where the coefficient $\bar{M}_{\ell m n}$ of $\{,\}^{\bar{M}}$ is the complex conjugate of $M_{\ell m n}$. If we use a simple symmetric difference operator as $\Delta$, the doubling problem is generated. To avoid the problem, we can introduce a supersymmetric Wilson term by replacing a mass $m$ to $m \delta_{m, n}+H_{m, n}$, where

$$
H_{m, n} \equiv r \frac{\delta_{m+1, n}+\delta_{m-1, n}-2 \delta_{m, n}}{2}
$$

with a Wilson parameter $r$. This action is exactly invariant under the subset transformation

$$
\left(\delta_{+}, \delta_{-}\right)
$$

and its algebra is

$$
\left\{\delta_{+}, \delta_{-}\right\}=\delta_{ \pm}^{2}=0 .
$$

Actually, under this transformation (3.6), it is shown that the action (3.4) is invariant

$$
\begin{aligned}
\delta_{+} S= & i \lambda\left(\left\{\bar{\chi}_{+}, \phi_{+}\right\}^{M}, F_{+}\right)+i \lambda\left(\left\{\phi_{+}, \bar{\chi}_{+}\right\}^{M}, F_{+}\right)-2 i \lambda\left(\left\{\bar{\chi}_{+}, \bar{\chi}_{+}\right\}^{M}, \chi_{+}\right) \\
& -2 i \lambda\left(\left\{\bar{\chi}_{+}, \phi_{+}\right\}^{M}, F_{+}\right)+i \lambda^{*}\left(\left\{\phi_{-}, \phi_{-}\right\}^{\bar{M}},-i \Delta \bar{\chi}_{-}\right)-2 i \lambda^{*}\left(\left\{\bar{\chi}_{-}, \phi_{-}\right\}^{\bar{M}}, i \Delta \phi_{-}\right)=0 \\
\delta_{-} S= & i \lambda\left(\left\{\phi_{+}, \phi_{+}\right\}^{M},-i \Delta \bar{\chi}_{+}\right)-2 i \lambda\left(\left\{\bar{\chi}_{+}, \phi_{+}\right\}^{M}, i \Delta \phi_{+}\right) \\
& +i \lambda^{*}\left(\left\{\bar{\chi}_{-}, \phi_{-}\right\}^{\bar{M}}, F_{-}\right)+i \lambda^{*}\left(\left\{\phi_{-}, \bar{\chi}_{-}\right\}^{\bar{M}}, F_{-}\right)-2 i \lambda^{*}\left(\left\{\bar{\chi}_{-}, \bar{\chi}_{-}\right\}^{\bar{M}}, \chi_{-}\right) \\
& -2 i \lambda^{*}\left(\left\{\bar{\chi}_{-}, \phi_{-}\right\}^{\bar{M}}, F_{-}\right)=0
\end{aligned}
$$

where we used CLR (2.6) the symmetric property of the inner product and the field product rule.

The subset (3.6) of the full SUSY (3.1) can be expressed by superfield formulation by introducing Grassmann numbers, $\theta_{+}, \theta_{-}$with $\theta_{ \pm}^{2}=0$. We define

$$
\begin{aligned}
\Phi_{+n} & \equiv \phi_{+n}+\theta_{+} \bar{\chi}_{+n} \\
\Upsilon_{+n} & \equiv F_{+n}-i \theta_{-}\left(\Delta \bar{\chi}_{+}\right)_{n} \\
\Psi_{+n} & \equiv \chi_{+n}+\theta_{+} F_{+n}+i \theta_{-}\left(\Delta \Phi_{+}\right)_{n} \\
S_{+n} & \equiv \bar{\chi}_{+n} \\
\Phi_{-n} & \equiv \phi_{-n}+\theta_{-} \bar{\chi}_{-n} \\
\Upsilon_{-n} & \equiv F_{-n}-i \theta_{+}\left(\Delta \bar{\chi}_{-}\right)_{n} \\
\Psi_{-n} & \equiv \chi_{-n}+\theta_{+} F_{-n}+i \theta_{+}\left(\Delta \Phi_{-}\right)_{n} \\
S_{-n} & \equiv \bar{\chi}_{-n} .
\end{aligned}
$$

Combining (3.6) and (3.9), the transformation for the superfields can be written as

$$
\begin{aligned}
& \delta_{+} \Phi_{+n}=S_{+n}, \quad \delta_{-} \Phi_{+n}=0, \\
& \delta_{+} \Phi_{-n}=0, \quad \delta_{-} \Phi_{-n}=S_{-n}, \\
& \delta_{+} \Psi_{+n}=\Upsilon_{+n}, \quad \delta_{-} \Psi_{+n}=i\left(\Delta \Phi_{+}\right)_{n}, \\
& \delta_{+} \Psi_{-n}=i\left(\Delta \Phi_{-}\right)_{n}, \quad \delta_{-} \Psi_{-n}=\Upsilon_{-n}, \\
& \delta_{+} \Upsilon_{+n}=0, \quad \delta_{-} \Upsilon_{+n}=-i\left(\Delta S_{+}\right)_{n}, \\
& \delta_{+} \Upsilon_{-n}=-i\left(\Delta S_{-}\right)_{n}, \quad \delta_{-} \Upsilon_{-n}=0 \text {, } \\
& \delta_{+} S_{+n}=0, \quad \delta_{-} S_{+n}=0, \\
& \delta_{+} S_{-n}=0, \quad \delta_{-} S_{-n}=0 .
\end{aligned}
$$


The transformation (3.10) implies

$$
\delta_{ \pm} F\left(\Phi_{ \pm}, \cdots\right)=\frac{\partial}{\partial \theta_{ \pm}} F\left(\Phi_{ \pm}, \cdots\right)
$$

where $F\left(\Phi_{ \pm}, \cdots\right)$ means arbitrary function of superfields (3.9). The action (3.4) is rewritten as

$$
\begin{aligned}
S= & \int d^{2} \theta\left(\Psi_{-}, \Psi_{+}\right)+i \int d^{2} \theta \theta_{-}\left(\Phi_{+}, m \Psi_{+}\right)+i \lambda \int d^{2} \theta \theta_{-}\left(\left\{\Phi_{+}, \Phi_{+}\right\}^{M}, \Psi_{+}\right) \\
& +i \int d^{2} \theta \theta_{-}\left(\Phi_{-}, m^{*} \Psi_{-}\right)+i \lambda^{*} \int d^{2} \theta \theta_{+}\left(\left\{\Phi_{-}, \Phi_{-}\right\}^{\bar{M}}, \Psi_{-}\right) .
\end{aligned}
$$

where $\int d^{2} \theta \theta_{-} \theta_{+}=1$ is used. We explicitly verify that the action (3.12) is invariant under (3.6). Considering a Wilson term $H$ in $m$, the following idenities and CLRs

$$
\begin{gathered}
\left(\Phi_{ \pm}, \Delta \Phi_{ \pm}\right)=\left(\Phi_{ \pm}, H \Delta \Phi_{ \pm}\right)=0, \\
\left(\left\{\Phi_{+}, \Phi_{+}\right\}^{M}, \Delta \Phi_{+}\right)=\left(\left\{\Phi_{-}, \Phi_{-}\right\}^{\bar{M}}, \Delta \Phi_{-}\right)=0,
\end{gathered}
$$

the invariance of the action 3.12

$$
\begin{aligned}
\delta_{+} S= & \int d^{2} \theta \delta_{+}\left(\Psi_{-}, \Psi_{+}\right)-i \int d^{2} \theta \theta_{-}\left(\delta_{+}\left(\Phi_{+}, m \Psi_{+}\right)+\lambda \delta_{+}\left(\left\{\Phi_{+}, \Phi_{+}\right\}^{M}, \Psi_{+}\right)\right. \\
& +i \int d^{2} \theta \theta_{+}\left(-\left(\Phi_{-}, i m^{*} \Delta \Phi_{-}\right)-\lambda^{*}\left(\left\{\Phi_{-}, \Phi_{-}\right\}^{\bar{M}}, i \Delta \Phi_{-}\right)\right) \\
= & \int d^{2} \theta \frac{\partial}{\partial \theta_{+}}\left(\Psi_{-}, \Psi_{+}\right)-i \int d^{2} \theta \theta_{-}\left(\frac{\partial}{\partial \theta_{+}}\left(\Phi_{+}, m \Psi_{+}\right)+\lambda \frac{\partial}{\partial \theta_{+}}\left(\left\{\Phi_{+}, \Phi_{+}\right\}^{M}, \Psi_{+}\right)\right)=0 \\
\delta_{-} S= & \int d^{2} \theta \delta_{-}\left(\Psi_{-}, \Psi_{+}\right)-i \int d^{2} \theta \theta_{+}\left(\delta_{-}\left(\Phi_{-}, m^{*} \Psi_{-}\right)+\lambda^{*} \delta_{-}\left(\left\{\Phi_{-}, \Phi_{-}\right\}^{\bar{M}}, \Psi_{-}\right)\right) \\
& +i \int d^{2} \theta \theta_{-}\left(-\left(\Phi_{+}, i m \Delta \Phi_{+}\right)-\lambda\left(\left\{\Phi_{+}, \Phi_{+}\right\}^{M}, i \Delta \Phi_{+}\right)\right. \\
= & \int d^{2} \theta \frac{\partial}{\partial \theta_{-}}\left(\Psi_{-}, \Psi_{+}\right)-i \int d^{2} \theta \theta_{+}\left(\frac{\partial}{\partial \theta_{-}}\left(\Phi_{-}, m^{*} \Psi_{-}\right)+\lambda^{*} \frac{\partial}{\partial \theta_{-}}\left(\left\{\Phi_{-}, \Phi_{-}\right\}^{\bar{M}}, \Psi_{-}\right)\right) \\
= & 0
\end{aligned}
$$

is shown.

\section{Supersymmetry, Mass degeneracy and Nicolai mappings}

In applying supersymmetry to particle physics, it is necessary for the mass degeneracy between boson and fermion in the symmetry limit. The degeneracy is clarified by using Ward-Takahashi relations by the subset supersymmetry (3.6). From $\delta_{+}\left\langle\phi_{+m} \chi_{-n}\right\rangle=0$ and $\delta_{-}\left\langle\phi_{-m} \chi_{+n}\right\rangle=0$, the following two relations

$$
\begin{aligned}
& \left\langle\phi_{+m}\left(\Delta \phi_{-}\right)_{n}\right\rangle=i\left\langle\bar{\chi}_{+m} \chi_{-n}\right\rangle \\
& \left\langle\phi_{-m}\left(\Delta \phi_{+}\right)_{n}\right\rangle=i\left\langle\bar{\chi}_{-m} \chi_{+n}\right\rangle
\end{aligned}
$$

are derived. We can calculate boson's and fermion's masses $\left(m_{b}, m_{f}\right)$ by taking the limit $|m-n| \rightarrow$ $\infty$ of two-point functions,

$$
\lim _{|n-m| \rightarrow \infty}\left\langle\phi_{+m} \phi_{-n}\right\rangle=K_{b} e^{-m_{b}|n-m|}
$$


and

$$
\lim _{|n-m| \rightarrow \infty}\left\langle\bar{\chi}_{+m} \chi_{-n}\right\rangle=K_{f} e^{-m_{f}|n-m|},
$$

with $K_{b}$ and $K_{f}$ are constants. In the case of a symmetric difference operator, we can explicitly calculate

$$
\begin{gathered}
\lim _{m-n \rightarrow \infty}\left\langle\phi_{+m}\left(\Delta \phi_{-}\right)_{n}\right\rangle=-\left(\sinh m_{b}\right) K_{b} e^{-m_{b}(m-n)}, \\
\lim _{m-n \rightarrow \infty}\left\langle\bar{\chi}_{+m} \chi_{-n}\right\rangle=K_{f} e^{-m_{f}(m-n)} .
\end{gathered}
$$

Therefore, from 4.10 we can obtain

$$
-i\left(\sinh m_{b}\right) K_{b}=K_{f}, m_{b}=m_{f} .
$$

This result implies the exact mass degeneracy between fermion and boson.

Another result by our CLR is the existence of two kinds of local Nicolai mappings. In the existence, CLR plays a very important role as follows. After eliminating auxiliary fields $F_{ \pm}$, the action is reduced to

$$
\begin{aligned}
S^{\prime}= & \left(\Delta \phi_{-}, \Delta \phi_{+}\right)-i\left(\Delta \bar{\chi}_{-}, \chi_{+}\right)-i\left(\Delta \bar{\chi}_{+}, \chi_{-}\right)+i\left(\bar{\chi}_{+}, m \chi_{+}\right)+i\left(\bar{\chi}_{-}, m^{*} \chi_{-}\right) \\
& +2 i \lambda\left(\left\{\bar{\chi}_{+}, \phi_{+}\right\}^{M}, \chi_{+}\right)+2 i \lambda^{*}\left(\left\{\bar{\chi}_{-}, \phi_{-}\right\}^{\bar{M}}, \chi_{-}\right) \\
& +\left(m^{*} \phi_{-}+\lambda^{*}\left\{\phi_{-}, \phi_{-}\right\}^{\bar{M}}, m \phi_{+}+\lambda\left\{\phi_{+}, \phi_{+}\right\}^{M}\right.
\end{aligned}
$$

For this action, we can define two Nicolai mappings

$$
\begin{aligned}
& \xi_{+n} \equiv\left(\Delta \phi_{-}\right)_{n}+i m \phi_{+n}+i \lambda\left\{\phi_{+}, \phi_{+}\right\}_{m}^{M} \\
& \xi_{-n} \equiv\left(\Delta \phi_{+}\right)_{n}+i m^{*} \phi_{-n}+i \lambda^{*}\left\{\phi_{-}, \phi_{-}\right\}_{n}^{\bar{M}}
\end{aligned}
$$

and

$$
\begin{aligned}
& \xi_{+n}^{\prime} \equiv-\left(\Delta \phi_{-}\right)_{n}+i m \phi_{+n}+i \lambda\left\{\phi_{+}, \phi_{+}\right\}_{n}^{M} \\
& \xi_{-n}^{\prime} \equiv-\left(\Delta \phi_{+}\right)+i m^{*} \phi_{-n}+i \lambda^{*}\left\{\phi_{-}, \phi_{-}\right\}_{n}^{\bar{M}}
\end{aligned}
$$

To write down the bosonic part $S_{b}^{\prime}$ of $S^{\prime}$ as

$$
S_{b}^{\prime}=\sum_{n} \xi_{-n} \xi_{+n}=\sum_{n} \xi_{-n}^{\prime} \xi_{+n}^{\prime}
$$

it is necessary for vanishing cross terms(surface terms) conditions

$$
\left(\Delta \phi_{-}, m^{*} \phi_{-}+\lambda^{*}\left\{\phi_{-}, \phi_{-}\right\}^{\bar{M}}\right)=\left(\Delta \phi_{+}, m \phi_{+}+\lambda\left\{\phi_{+}, \phi_{+}\right\}^{M}\right)=0 .
$$

These conditions just correspond to CLR and a symmetric difference operator for any $\phi_{ \pm}$. The mappings are useful in calculating quantum effects in this model. The fermion part of the action in the model is written as

$$
S_{f}=\sum_{m n}\left(\bar{\chi}_{+m}, \bar{\chi}_{-m}\right)\left(\begin{array}{cc}
i m \delta_{m n}+2 i \lambda M_{n m k} \phi_{+k} & -i \Delta_{n m} \\
-i \Delta_{n m} & i m^{*} \delta_{m n}+2 i \lambda \lambda^{*} \bar{M}_{n m k} \phi_{-k}
\end{array}\right)\left(\begin{array}{c}
\chi_{+n} \\
\chi_{-n}
\end{array}\right) .
$$


When these fermions are transformed as the following way

$$
\left(\begin{array}{c}
\chi_{+n}, \\
\chi_{-n}, \\
\bar{\chi}_{+n}, \\
\bar{\chi}_{-n}
\end{array}\right) \rightarrow\left(\begin{array}{c}
\chi_{+n}^{\prime}, \\
\chi_{-n}^{\prime}, \\
\bar{\chi}_{+n}^{\prime}, \\
\bar{\chi}_{-n}^{\prime}
\end{array}\right)=\left(\begin{array}{c}
\chi_{+n}, \\
-\chi_{-n}, \\
\bar{\chi}_{+n}, \\
-\bar{\chi}_{-n}
\end{array}\right),
$$

the signs of the fermionic kinetic terms in the action (4.7) are changed and the fermion determinant is unchanged in any lattice size because the Jacobian is $(-1)^{2 N_{L}}$. The two descriptions $\left(\chi_{+}, \cdots\right)$ and $\left(\chi_{+}^{\prime}, \cdots\right)$ of the fermion corresponds to two Nicolai mappings.

\section{Summary}

We have constructed an $N=2$ supersymmetric quantum mechanical model on lattice. Although the realization of the supersymmetry in the model is a subset of full SUSY, the WardTakahashi identities lead to the excat degeneracy between fermion and boson. The new type of superfield formalism can describe the action and the transformation very concisely. Surprisingly, our model has a nonrenormalizaion theorem with respect to mass and interaction terms. The detail analysis will appear in our forthcoming paper [6].

To extend to higher-dimensional theories, we must reconsider CLR in higher dimensions carefully. As discussed in Ref.[3], it is difficult to maintain locality in a higher-dimensional extension of the CLR.

\section{References}

[1] M. Kato, M. Sakamoto, and H. So, Taming the Leibniz Rule on the Lattice, JHEP0805(2008)057.

[2] G. Bergner, Complete supersymmetry on the lattice and a No-Go theorem, JHEP1001(2010)024.

[3] M. Kato, M. Sakamoto, and H. So, A criterion for lattice supersymmetry: cyclic Leibniz rule, JHEP1305(2013)089.

[4] M. Beccaria, G. Curci, and E. D'Ambrosio, Simulation of supersymmetric models with a local Nicolai map, Phys. Rev. D58(1998)065009.

[5] S. Catterall, and E. Gregory, A Lattice path integral for supersymmetric quantum mechanics, Phys. Lett. B478(2000)349.

[6] M. Kato, M. Sakamoto, and H. So, in preparation. 